\title{
Der Begriff des Postponierers im Licht von Sprachvergleichsdaten Deutsch-Italienisch
}

\begin{abstract}
Der vorliegende Aufsatz untersucht die Syntax und Semantik sogenannter Postponierer, d.h. konjunktionaler Konnektoren, die den von ihnen eingeleiteten Nebensatz dem Hauptsatz stets nachstellen. Anhand von sodass und zumal werden die Kerneigenschaften solcher Konnektoren im Deutschen vorgestellt. Am Beispiel der italienischen Konjunktionen cosicché, tanto più che und perché wird diskutiert, ob der Begriff des Postponierers für den Sprachvergleich genutzt werden kann. In einem nächsten Schritt werden die Postponierer des Deutschen unter Beiziehung sprachgeschichtlicher Argumente präziser beschrieben und im Übergangsfeld zwischen Adverbkonnektoren und Subjunktoren verortet. Es zeigt sich, dass die untersuchten Konnektoren sich letztlich sehr unterschiedlich verhalten, sodass es fraglich erscheint, ob ihre Zusammenfassung zu einer gemeinsamen Klasse gerechtfertigt ist.

This article studies the syntax and semantics of so-called postponers, i.e. appending conjunctions which require the subordinate clause they introduce to follow the main clause. German sodass and zumal are prototypical examples, which illustrate the grammatical properties of those connectives. Other languages have conjunctions with similar characteristics, e.g. Italian cosicché, tanto più che and perché. In the first part of the article we discuss whether the concept of the postponer brings benefits for comparative grammatical descriptions. In a subsequent step, the syntactic and semantic behaviour of the German postponers is examined more thoroughly. Historical evidence characterises them as manifestations of the transition from adverbial connectives to subordinating conjunctions. It becomes apparent that each postponer has its individual grammatical properties, so that it seems questionable whether they should be considered instances of one and the same category.
\end{abstract}

\section{Einleitung}

Der Begriff Postponierer, der in diesem Aufsatz ${ }^{1}$ diskutiert wird, wurde erstmals von Pasch (2000, S. 25) eingeführt und in Pasch et al. (2003, S. 418f.) wiederaufgenommen. $\mathrm{Zu}$ den Postponierern werden konjunktionale Konnektoren wie sodass und zumal gezählt. Die durch sie eingeleiteten Teilsätze in Verknüpfungen wie (1) und (2) müssen dem anderen Teilsatz stets nachgestellt werden:

(1) In Ermangelung von Tierärzten gab es kein Netz zur epidemiologischen Überwachung, sodass sich die Seuche verbreitete. ${ }^{2}$

1 Der Aufsatz wurde während eines durch die Alexander-von-Humboldt-Stiftung (Aktenzeichen ITA 1148681 STP) geförderten Forschungsaufenthaltes von Miriam Ravetto am Institut für Deutsche Sprache in Mannheim (Mai bis August 2014) erarbeitet. Für die Inhalte sind die Autoren gemeinsam verantwortlich. Miriam Ravetto hat die Kapitel 1 und 2, Hardarik Blühdorn die Kapitel 3 und 4 verfasst. Eine frühere Version wurde am 2. Dezember 2014 von Miriam Ravetto in einem Gastvortrag am IDS vorgestellt. Die Autoren danken Patrick Brandt, Silvia Kutscher, Stefan Rabanus, Anna Volodina, Bernd Wiese, Angelika Wöllstein, Gisela Zifonun sowie zwei anonymen DS-Gutachtern für Diskussionsbeiträge und wertvolle Hinweise, der Alexander-von-Humboldt-Stiftung für die Förderung und dem Direktor des IDS, Prof. Dr. Ludwig M. Eichinger, für jederzeitige Unterstützung.

2 Die in diesem Aufsatz verwendeten Beispiele wurden mit der Suchmaschine Google auf deutsch- bzw. italienischsprachigen Internetseiten der Domänen .de und .it gefunden. Einzelne Beispiele wurden in Anlehnung an Internetbelege selbst gebildet (zur Arbeit mit Originalbelegen vgl. auch Blühdorn/Ravetto 2014, S. 39f.). 
(2) Die hohe Arbeitslosigkeit in der Europäischen Union bereitet uns allen Sorgen, zumal die rückläufige Tendenz anscheinend nicht anhält.

Eine Voranstellung des sodass- bzw. zumal-Satzes wie in (1a) und (2a) ist nicht möglich:

(1a) *Sodass sich die Seuche verbreitete, gab es in Ermangelung von Tierärzten kein Netz zur epidemiologischen Überwachung.

(2a) *Zumal die rückläufige Tendenz anscheinend nicht anhält, bereitet die hohe Arbeitslosigkeit in der Europäischen Union uns allen Sorgen.

Durch ihr Stellungsverhalten unterscheiden sich Postponierer von Subjunktoren. Subjunktoreingeleitete Nebensätze können dem anderen (syntaktisch übergeordneten) Teilsatz nachgestellt werden oder dessen Vorfeld einnehmen:

(3) Der Frosch tauchte unter, sobald er das Versprechen erhalten hatte.

(3a) Sobald er das Versprechen erhalten hatte, tauchte der Frosch unter.

In der älteren Literatur wurden Postponierer nicht als eigenständige syntaktische Klasse geführt, sondern den Subjunktoren zugerechnet. Dass manche Subjunktoren nur nachgestellte Sätze einleiten, ist aber schon seit langem bekannt. Nach Behaghel (1932, S. 264f.) waren in der Sprachgeschichte des Deutschen die meisten Nebensätze ursprünglich nachgestellt. Das gilt zum einen für „Sätze, die eine notwendige Ergänzung des Hauptsatzes enthalten“ (in heutiger Terminologie Subjekt- und Objektsätze), ferner für „Folgesätze, Absichtssätze“ (Konsekutiv- und Finalsätze), „Sätze, deren Zeit nach der des Hauptsatzes fällt“ (eine Teilgruppe der Temporalsätze), schließlich „Sätze mit Konjunktionen, die durch Ersparung von daz entstanden sind" (Behaghel nennt Sätze mit indem, nachdem und weil, also Instrumentalsätze, weitere Temporalsätze und Kausalsätze) und ,Sätze mit danne nach Komparativ und ihre Fortsetzungen mit als - wie" (Vergleichssätze). Alle diese Arten von Nebensätzen wurden nach Behaghel dem übergeordneten Satz in den älteren Sprachstufen durchweg nachgestellt. Erst in der jüngeren Sprachgeschichte seien solche Sätze auch in die Voranstellung getreten, und zwar insbesondere um Abfolgeverhältnisse ikonisch wiedergeben zu können. Lediglich Konsekutiv- und Vergleichssätze hätten ,ihre Stellung nach dem Hauptsatz" durchweg beibehalten. Nach Behaghel ist also das Stellungsverhalten, das in der Gegenwartssprache die Postponierer kennzeichnet, das sprachgeschichtlich ältere und das der Subjunktoren das jüngere. Dass „ursprünglich nur vorstehende Sätze hinter den Hauptsatz wandern", ist nach Behaghel (ebd., S. 264) "spärlich bezeugt".

Auf die Beschränkungen im Stellungsverhalten einiger subordinierender Konjunktionen (insbesondere der konsekutiven) wird auch in vielen anderen Grammatiken hingewiesen, etwa bei Helbig/Buscha (1972, S. 421), Boettcher/Sitta (1972, S. 144), Engel (1988, S. 725) und Zifonun/Hoffmann/Strecker (1997, S. 2311).

Pasch et al. (2003, S. 347ff.) unterteilen die Konnektoren in zwei Hauptgruppen: konnektintegrierbare (adverbiale) und nichtkonnektintegrierbare (konjunktionale). Beide Gruppen werden noch weiter subklassifiziert. Zu den konjunktionalen Konnektoren gehören:

(i) Konjunktoren

(ii) Verbzweitsatz(V2)-Einbetter

(iii) Postponierer

(iv) Subjunktoren 
Nach Pasch et al. (2003, S. 352) kann jede dieser Klassen durch zwei syntaktische Merkmale gekennzeichnet werden, die folgendermaßen verteilt sind:

\begin{tabular}{|l|c|c|c|c|}
\hline & Konjunktoren & V2-Einbetter & Postponierer & Subjunktoren \\
\hline subordinierend & - & - & + & + \\
\hline einbettend & - & + & - & + \\
\hline
\end{tabular}

Tab. 1: Verteilung syntaktischer Eigenschaften auf die Klassen der konjunktionalen Konnektoren (nach Pasch et al. 2003)

Als subordiniert gelten hier Teilsätze mit Verbletztstellung; als eingebettet (integriert) gelten Teilsätze, die ins Vorfeld des einbettenden Satzes treten können. Der durch einen konjunktionalen Konnektor eingeleitete Satz wird als internes Konnekt des Konnektors bezeichnet. Postponierer bilden mit ihrem internen Konnekt eine Postponiererphrase. Diese darf laut Pasch et al. (ebd., S. 419), ,nicht vor dem externen Konnekt" stehen, d.h. sie muss dem übergeordneten Teilsatz nachgestellt werden.

Der Begriff Postponierer steht bei Pasch et al. (ebd., S. 352) für eine klassifikatorische Kategorie einer deskriptiven Grammatik des Deutschen, und zwar für eine „Übergangsklasse zwischen Subjunktoren und Konjunktoren". Auch von anderen Autoren ist vorgeschlagen worden, das Feld der Satzverknüpfungen als Kontinuum zu konzeptualisieren, in dem mehr als zwei syntaktische Verfahren unterschieden werden können (vgl. Lehmann 1988, S. 184ff.; König/van der Auwera 1990, S. 343; Kortmann 1996, S. 56ff.; Johannessen 1998, S. 237ff.; Cristofaro 2003, S. 22ff. u.v.m.). Der eine Pol einer solchen Skala ist dadurch gekennzeichnet, dass zwei Ausdrücke strukturell gleichrangig nebeneinanderstehen. Ihm entspricht die asyndetische (im Sinne von Pasch et al. 2003, S. 305 parataktische) Reihung: Zwei voneinander unabhängige Ausdrücke folgen ohne syntaktische Verknüpfung aufeinander. Den anderen Pol der Skala bilden Verknüpfungen, in denen ein Ausdruck vollständig als Konstituente in den anderen integriert ist. Zwischen asyndetischer Reihung und vollständiger Integration liegen Grade unvollständiger Integration. In Verknüpfungen mit Konjunktoren (koordinierenden Konjunktionen) ist das linear zweite Konnekt enger mit dem Konjunktor verbunden als das erste (vgl. Johannessen 1998, S. 108ff.; Blühdorn 2008; Blühdorn/Ravetto 2014, S. 4). Das kann als ein erster Hinweis auf syntaktische Unselbständigkeit des zweiten Konnekts gedeutet werden. Nach Quirk et al. (1985, S. 927) bilden die sogenannten conjuncts (Adverbkonnektoren wie yet, so, nor oder however) im Englischen eine syntaktische Klasse zwischen Konjunktoren und Subjunktoren. Auch Postponierer stehen nach Pasch et al. (2003) auf der Skala zwischen diesen beiden Klassen.

Die Konstituentenstruktur einer Postponierer-Verknüpfung kann folgendermaßen veranschaulicht werden (vgl. Pasch et al. 2003, S. 419; Blühdorn 2008; Breindl/Volodina/ Waßner 2014, S. 25): 


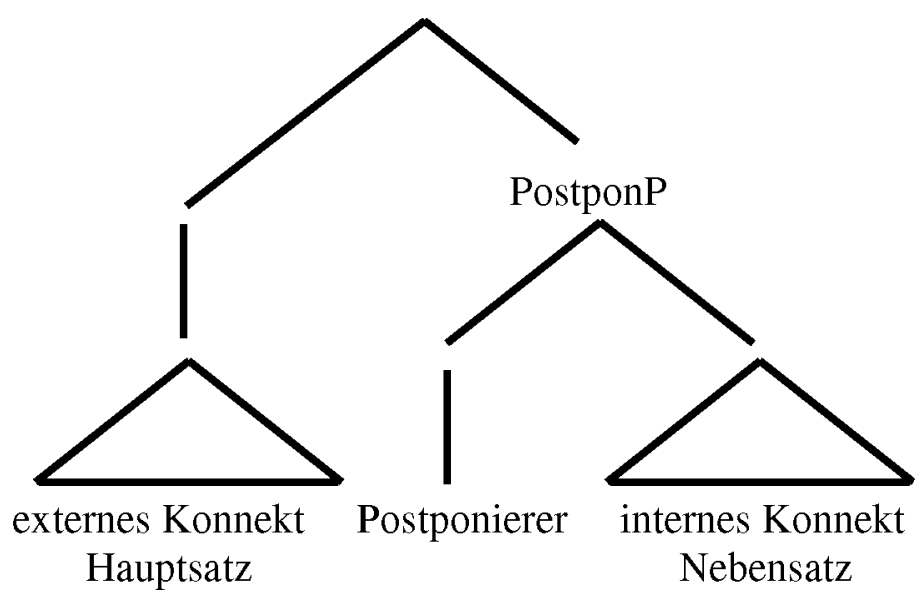

Abb. 1: Konstituentenstruktur einer Postponierer-Verknüpfung

Der Postponierer bildet eine Konstituente mit seinem internen Konnekt; die Postponiererphrase bildet eine komplexere Konstituente mit dem externen Konnekt. Die syntaktische Natur der Verbindung zwischen der Postponiererphrase und dem externen Konnekt ist damit aber noch nicht vollständig aufgeklärt. Wenn man die Struktur des Gesamtsatzes nach dem X-bar-Schema darstellt (vgl. z.B. Lohnstein 2014) und Postponiererphrasen als adverbiale Adjunkte auffasst, kommen für sie zwei Strukturpositionen in Betracht: die rechten Adjunktpositionen zur IP oder zur CP (vgl. Wöllstein 2010, S. 82ff.; Blühdorn/ Ravetto 2014, S. 13ff.):

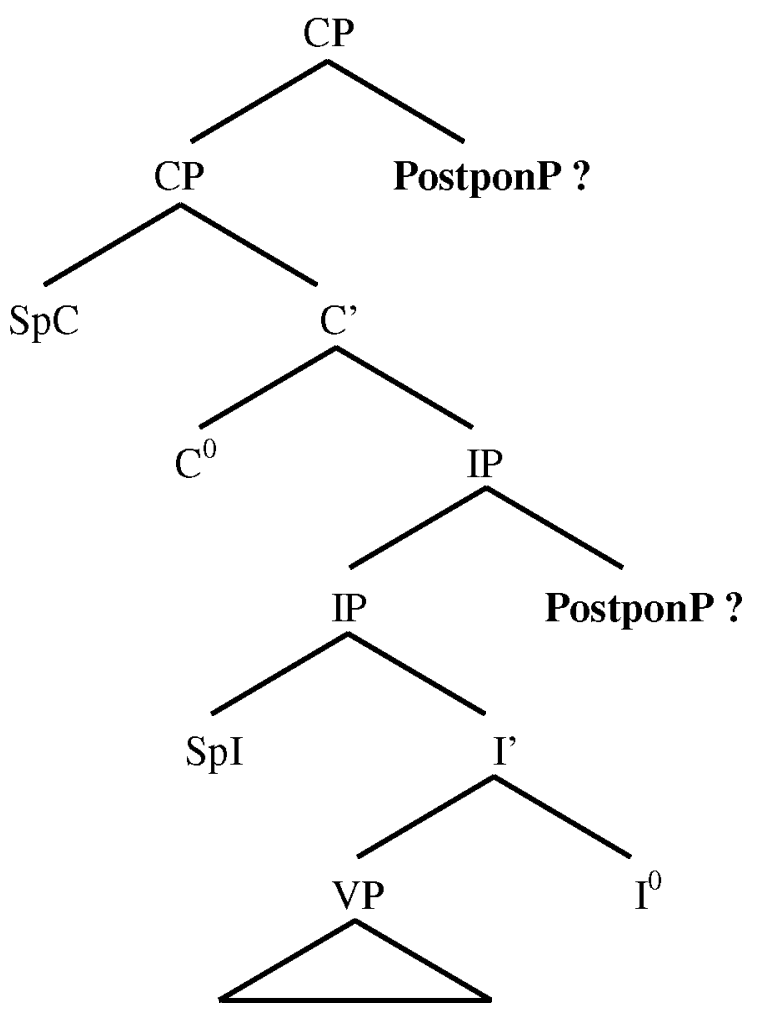

Abb. 2: Mögliche Strukturpositionen der Postponiererphrase 
Als Adjunkt zur IP würde die Postponiererphrase in dem Teil des Satzes stehen, der nach dem Felderschema (vgl. Wöllstein 2010, S. 22) als Nachfeld bezeichnet wird. So wird ihre Position bei Zifonun/Hoffmann/Strecker (1997, S. 2311) gedeutet. Als Adjunkt zur CP wäre die Postponiererphrase syntaktisch desintegriert. Sie stünde im rechten Außenfeld des Satzes (der rechten externen Adjunktposition REA im Sinne von Blühdorn 2012, S. 204). Blühdorn/Ravetto (2014, S. 8ff.) argumentieren, dass Nachfeldstellung weniger plausibel ist. Durch einen Subjunktor eingeleitete Sätze erlauben als Alternative zur Nachstellung die Voranstellung ins Vorfeld, also in eine syntaktische Integrationsposition. Für sie ist anzunehmen, dass sie bei Nachstellung das Nachfeld einnehmen und dort ebenfalls syntaktisch integriert sind. Postponierersätze können nicht das Vorfeld einnehmen. Das legt die Annahme nahe, dass sie syntaktisch desintegriert sind, also im rechten Außenfeld stehen.

Deutet man wie Holler (2007, S. 256; 2008, S. 207) und zahlreiche weitere Autoren die Möglichkeit der Variablenbindung zwischen den Teilsätzen eines Satzgefüges als Hinweis auf syntaktische Integration und ihre Unmöglichkeit als Hinweis auf syntaktische Desintegration, so scheinen auch Bindungsdaten dafür zu sprechen, dass Postponierersätze im Gegensatz zu Subjunktorsätzen syntaktisch desintegriert sind. So kann die Variable er in dem zumal-Satz in (4) nach unserem Sprachgefühl nicht durch den Quantifikator niemand gebunden werden. In einer Verknüpfung mit dem Subjunktor weil ist die Bindung dagegen problemlos:

(4) Niemand ${ }_{i}$ strengt sich an, weil/ ${ }^{*}$ zumal er $_{i}$ zeigen muss, was er ${ }_{i}$ kann.

Wenn man mehr Daten anschaut, zeigen sich die Verhältnisse aber uneinheitlich. So scheint die Bindung von er durch jeder Mann in der zumal-Verknüpfung in (5) unproblematisch zu sein:

(5) [Jeder Mann $]_{i}$ gilt als potenzieller Vergewaltiger, weil/zumal $\mathrm{er}_{i} \operatorname{sein}_{i}$ natürliches Werkzeug immer bei sich ${ }_{1}$ trägt.

(6) und (7) sind ein ähnliches Paar mit sodass. In (6) erscheint uns die Bindung von er durch niemand bei sodass-Verknüpfung fragwürdig. In der Verknüpfung mit dem finalen Subjunktor damit ist sie möglich. Dagegen ist in (7) die Bindung von er durch jeder Lernpartner anscheinend auch in der sodass-Verknüpfung unproblematisch. Sodass legt hier eine finale Deutung im Sinne von 'damit' nahe:

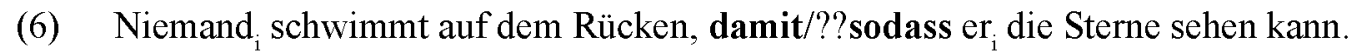

(7) [Jeder Lempartner $]_{i}$ dokumentiert seinen ${ }_{i}$ Lern- und Lebensweg, damit/sodass er ${ }_{i}$ jederzeit über seine ${ }_{i}$ Erfolge berichten kann.

Die Möglichkeiten der Variablenbindung in Adverbialsatzgefügen sind oft schwer beurteilbar und empirisch noch zu wenig untersucht. Ihre Aussagekraft in Bezug auf syntaktische Integration ist noch nicht vollständig aufgeklärt (zur Diskussion vgl. Christ 2014, S. 139ff.). Wir interpretieren die Beobachtungen an den obigen Satzpaaren so, dass der zumal- bzw. sodass-Satz, wenn keine Variablenbindung möglich ist, im rechten Außenfeld, wenn sie möglich ist, im Nachfeld steht. Eventuell kann man die Daten im Sinne des von Behaghel beschriebenen sprachhistorischen Prozesses in der Weise deuten, dass zumal und sodass gegenwärtig die Fähigkeit annehmen, von ihnen eingeleitete nachgestellte Nebensätze syntaktisch zu integrieren. Auch wenn eine Voranstellung der Nebensätze noch nicht möglich ist, werden zumal und sodass durch die syntaktische Integrationsfähigkeit prototypischen Subjunktoren ähnlicher. Bei sodass deutet sich in Fällen wie (7) an, 
dass mit dem Wandel zum Subjunktor eine semantische Veränderung zu einer finalen Bedeutung einhergeht.

Für sprachvergleichende Untersuchungen auf dem Gebiet der Konnektoren (wie z.B. Ravetto/Blühdorn 2011; Blühdorn/Ravetto 2014; Ravetto/Blühdorn in Vorb.) stellt sich die Frage, ob Kategorien wie die des Postponierers nur für die Beschreibung des Deutschen als Einzelsprache oder auch sprachübergreifend anwendbar sind. Sprachübergreifend anwendbare Kategorien können als tertium comparationis dienen. Einige Konnektoren des Italienischen, z.B. cosicché ('sodass', 'damit') und tanto più che ('umso mehr als', 'auch weil', 'zumal'), zeigen ein ähnliches Stellungsverhalten wie die deutschen Postponierer. Damit könnte die Kategorie des Postponierers auch für die Grammatik des Italienischen verwendbar sein.

In Kapitel 2 dieses Aufsatzes betrachten wir zunächst einige italienische Daten. Im Anschluss daran stellen wir in Kapitel 3 für das Deutsche die Frage, wie die Grammatik der Postponierer, insbesondere unter dem Gesichtspunkt der syntaktischen Integration, zu präzisieren ist. Dabei werden wir auch diachrone Gesichtspunkte berücksichtigen. In Kapitel 4 werden die Ergebnisse zusammengefasst und interpretiert.

\section{Postponierer im Italienischen?}

\subsection{Subordination vs. Koordination}

Eine den deutschen Postponierern entsprechende syntaktische Klasse von Konnektoren ist für das Italienische bisher nicht postuliert worden. In den italienischen Grammatiken wird allgemein nur zwischen subordinierenden und koordinierenden Konjunktionen (congiunzioni subordinanti und coordinanti) unterschieden (Serianni 1989, S. 529ff.; Renzi/Salvi/ Cardinaletti Bd. 1, 1988, S. 227ff., Bd. 2, 1991, S. 633ff. u.v.m.). Ein klares Beispiel für eine subordinierende Konjunktion ist poiché ('weil', 'da'). In (8) ist das erste Konnekt (venderà tutto) syntaktisch übergeordnet; das zweite Konnekt (andrà in Brasile) ist der Nebensatz:

(8) Venderà tutto, poiché andrà in Brasile.

verkaufen-3sg-fut alles, weil gehen-3sg-fut nach Brasilien

'Er wird alles verkaufen, weil er nach Brasilien gehen wird.'3

Eine prototypische koordinierende Konjunktion des Italienischen ist $e$ ('und'); $e$-Verknüpfungen wie in (9) zeigen kein Unterordnungsverhältnis zwischen den Teilsätzen. Zwischen dem ersten Konnekt (venderà tutto) und dem zweiten (andrà in Brasile) besteht nur die syntaktische Asymmetrie, die für Koordinationen typisch ist (s.o. Kap. 1):

(9) Venderà tutto $\mathbf{e}$ andrà in Brasile.

verkaufen-3sg-fut alles und gehen-3sg-fut nach Brasilien

'Er wird alles verkaufen und (er wird) nach Brasilien gehen.'

Die syntaktische Unterscheidung zwischen subordinierten und nicht-subordinierten Sätzen kann im Italienischen nicht mit der Verbstellung in Zusammenhang gebracht werden. Unselbständige Sätze des Italienischen haben die gleiche SVO-Struktur wie selbständige Sätze. Das wichtigste Unterscheidungskriterium ist die Möglichkeit bzw. Unmöglichkeit, den Teilsatz voranzustellen (vgl. Renzi/Salvi/Cardinaletti Bd. 1, 1988, S. 288f.; Haspel-

\footnotetext{
3 Zu den italienischen Beispielen geben wir wörtliche Glossen und literarische Übersetzungen auf Deutsch.
} 
math 1995, S. 13f.). Ist Voranstellung möglich, so handelt es sich um einen Nebensatz; wird er durch eine Konjunktion eingeleitet wie in (8a), so ist diese ein Subjunktor:

(8a) Poiché andrà in Brasile, venderà tutto.

weil gehen-3sg-fut nach Brasilien, verkaufen-3sg-fut alles

'Weil er nach Brasilien gehen wird, wird er alles verkaufen.'

Ist keine Voranstellung möglich, so gelten die Teilsätze als koordiniert; ein konjunktionaler Einleiter ist dann ein Konjunktor. In einer $e$-Verknüpfung wie (9) ist zwar der Austausch der verknüpften Konnekte (eventuell mit Bedeutungsveränderung) möglich wie in (9a), aber die Voranstellung des zweiten Konnekts zusammen mit dem Konjunktor wie in (9b) ist ausgeschlossen:

(9a) Andrà in Brasile e venderà tutto.

gehen-3sg-fut nach Brasilien und verkaufen-3sg-fut alles

'Er wird nach Brasilien gehen und (er wird) alles verkaufen.'

(9b) *E andrà in Brasile venderà tutto.

und gehen-3sg-fut nach Brasilien verkaufen-3sg-fut alles

Ein weiteres Unterscheidungskriterium ist die Möglichkeit der Variablenbindung und der Koreferenz von Phorika (vgl. Renzi/Salvi/Cardinaletti Bd. 2, 1991, S. 749). Eine Variable im linear ersten Teilsatz, z.B. ein leeres Subjekt, kann durch einen Operator im zweiten Teilsatz, z.B. einen Quantifikator wie ognuno ('jeder') in (10a), nur dann gebunden werden, wenn der erste Teilsatz subordiniert ist. Ebenso können phorische Ausdrücke und leere Proformen im ersten Teilsatz nur bei Subordination koreferent mit Bezugsausdrücken im zweiten Teilsatz sein. So erlaubt (11a) eine Lesart, in der das leere Subjekt des siccome-Satzes koreferent mit dem expliziten Subjekt des zweiten Teilsatzes ist. Der seSatz in (10a) und der siccome-Satz in (11a) sind subordiniert und syntaktisch integriert; se und siccome sind Subjunktoren:

(10a) Se $\emptyset_{i}$ è lodato dal capo, ognuno ${ }_{i}$ è invogliato a fare un bel lavoro.

wenn $\emptyset_{i}$ sein-3sg-präs loben-partII vom Chef, jeder ${ }_{i}$ sein-3sg-präs motivieren-partII zu machen-inf eine gute Arbeit

'Wenn er ${ }_{i}$ vom Chef gelobt wird, ist jeder ${ }_{i}$ motiviert, eine gute Arbeit zu machen.'

(11a) Siccome $\emptyset_{i}$ non aveva dormito molto Mario ${ }_{i}$ era stanco.

da $\emptyset_{i}$ nicht schlafen-3sg-pqp viel Mario ${ }_{i}$ sein-3sg-prät müde

'Da er ${ }_{1}$ nicht viel geschlafen hatte, war Mario müde.'

Bei Koordination ist keine entsprechende Variablenbindung bzw. Koreferenz möglich. So erlaubt (10b) keine Lesart, in der das leere Subjekt des ersten Teilsatzes durch den Quantifikator ognuno im zweiten Teilsatz gebunden wird, und in (11b) kann das leere Subjekt des ersten Teilsatzes nicht koreferent mit dem expliziten Subjekt des zweiten sein. Die ersten Teilsätze in diesen Beispielen sind den zweiten weder subordiniert noch syntaktisch in sie integriert:

(10b) $* \emptyset_{i}$ è stato lodato dal capo e ognuno è invogliato a fare un bel lavoro

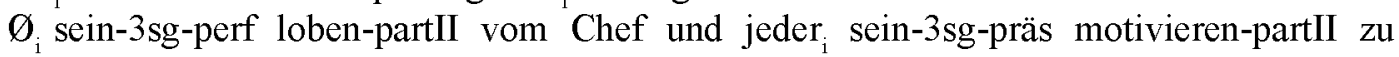
machen-inf eine gute Arbeit

(11b) $* \varnothing_{1}$ non aveva dormito molto e Mario era stanco $\emptyset_{i}$ nicht schlafen-3sg-pqp viel und Mario $_{i}$ sein-3sg-prät müde 
Die konjunktionalen Konnektoren cosicché und tanto più che scheinen sich in die Zweiteilung zwischen Subjunktoren und Konjunktoren nicht gut einzufügen. Nach Serianni (1989, S. 585) und Lombardi Vallauri (2000, S. 82ff.) müssen mit ihnen eingeleitete Sätze dem anderen Teilsatz stets nachgestellt werden, sind aber dennoch Nebensätze. Auch von Sätzen, die mit perché ('weil') eingeleitet sind, wird manchmal behauptet, sie müssten nachgestellt werden (vgl. z.B. Previtera 1996, S. 35; dazu: Ravetto/Blühdorn 2011, S. 218ff.). Im Folgenden untersuchen wir die Syntax dieser drei Konnektoren genauer.

\section{$2.2 \quad$ Cosicché}

Cosicché ('sodass') steht gegenwartssprachlich noch neben dem nicht univerbierten, semantisch gleichwertigen cosi che (vgl. Serianni 1989, S. 586; Schwarze 1995, S. 439, 743). ${ }^{4}$ In Beispielen wie (12) wäre eine Voranstellung des konsekutiven cosicché-Satzes unmöglich:

(12) La produzione avviene in grandi unità, cosicché i valori economici sono spesso più importanti della salute umana.

die Produktion sich vollziehen-3sg-präs in großen Einheiten, sodass die Werte wirtschaftlichen sein-3pl-präs oft mehr wichtige als die Gesundheit menschliche

'Die Produktion vollzieht sich in großen Einheiten, sodass die wirtschaftlichen Werte oft wichtiger sind als die Gesundheit der Menschen.'

(12a) *Cosicché i valori economici sono spesso più importanti della salute umana, la produzione avviene in grandi unità.

Nach Behaghel (1932, S. 263) ist in Konsekutivverknüpfungen des Deutschen die ältere Nachstellung des Nebensatzes aus semantischen Gründen obligatorisch geblieben (vgl. auch Zifonun/Hoffmann/Strecker 1997, S. 2311; Pittner 1999, S. 276f.; Konerding 2002, S. 12ff.). Die im Konsekutivsatz beschriebene Wirkung folgt der im Hauptsatz beschriebenen Ursache zeitlich immer nach. Die Nachstellung ist deshalb ikonisch. Gleiches gilt für konsekutive cosicché-Verknüpfungen. Ebenso wie beim deutschen sodass ist das Adverb così ('so') im Laufe der Sprachgeschichte aus dem vorangestellten Hauptsatz nach rechts an die Spitze des Konsekutivsatzes ,gewandert" und wurde dort mit dem Subordinationsanzeiger che univerbiert (vgl. Serianni 1989, S. 586).

Nach der in den italienischen Grammatiken üblichen Unterscheidung könnte die NichtVoranstellbarkeit des cosicché-Satzes zu der Schlussfolgerung führen, dass cosicché ein Konjunktor ist. Variable in konsekutiven cosicché-Sätzen können in der Regel nicht durch Quantifikatoren im vorausgehenden Teilsatz gebunden werden; in Verknüpfungen mit Subjunktoren wie quando ist die Bindung dagegen unproblematisch:

(13) [Nessuna produzione $]_{i}$ ha avuto grande successo, quando/* cosicché $\emptyset_{\mathrm{i}}$ è stata riproposta. [keine Produktion $]_{i}$ haben-3sg-präs haben-partII großen Erfolg, als/sodass $\emptyset_{i}$ sein-3sg-präs sein-partII wiederaufnehmen-partII

'[Keine Produktion $]_{i}$ hat großen Erfolg gehabt, als/*sodass sie $_{i}$ wiederaufgenommen wurde.'

${ }_{4}$ Ein weiteres Äquivalent des deutschen sodass ist sicché (vgl. Schwarze 1995, S. 743). Serianni (1989) unterscheidet zwischen sicché als congiunzione coordinante conclusiva ( $\mathrm{S}$. 541) und als congiunzione subordinante consecutiva (S. 586). 
Dennoch ordnen die Grammatiken des Italienischen cosicché mit Verweis auf seine Entstehung aus einem Adverb und einem Komplementierer in der Regel als Subjunktor ein (Serianni 1989, S. 585; Renzi/Salvi/Cardinaletti Bd. 2, 1991, S. 825ff.; Salvi/Vanelli 2004, S. 282f.). Diese Einordnung passt zu der Beobachtung, dass es Fälle gibt, in denen eine Variablenbindung in cosicché-Sätze hinein doch möglich ist:

(14) [Ogni libro $]_{i}$ verrà letto e toccato, cosicché $\emptyset_{i}$ potrà venir riconosciuto dal singolo bambino.

[jedes Buch $]_{i}$ kommen-3sg-fut lesen-partII und anfassen-partII, sodass $\emptyset_{\mathrm{i}}$ können-3sg-fut kommen-inf wiedererkennen-partII vom einzelnen Kind

'[Jedes Buch $]_{i}$ wird gelesen und angefasst werden, sodass es ${ }_{i}$ vom einzelnen Kind wiedererkannt werden kann.'

In (14) ist, ähnlich wie in dem deutschen sodass-Beispiel (7), zu beobachten, dass für cosicché eine finale Lesart im Sinne von affinché ('damit') möglich ist. In vielen Grammatiken des Italienischen wird übersehen, dass cosicché relativ häufig als Finalkonnektor verwendet wird. Das Verb des cosicché-Satzes erscheint dann typischerweise im Konjunktiv und/oder in einer modalen Periphrase, etwa mit potere ('können') (vgl. Schwarze 1995, S. 743f.):

(15) Proponiamo un insegnamento più ampio per i farmacisti, cosicché possano entrare più stabilmente in questo campo.

vorschlagen-1pl-präs eine Ausbildung mehr breit für die Apotheker, sodass können-3plkonj-präs eintreten-inf mehr stark in dieses Feld

'Wir schlagen eine breitere Ausbildung für die Apotheker vor, damit sie besser in dieses Feld einsteigen können.'

Anders als finale sodass-Sätze können finale cosicché-Sätze sogar vorangestellt werden:

(16) Cosicché le donne possano aiutare a liberare l'altra metà della razza umana, dobbiamo liberarle.

damit die Frauen können-3pl-konj-präs helfen-inf zu befreien-inf die andere Hälfte der Menschheit, müssen-lpl-präs befreien-inf sie

'Damit die Frauen dabei helfen können, die andere Hälfte der Menschheit zu befreien, müssen wir sie befreien.'

Ein vorangestellter cosicché-Satz muss stets final gelesen werden; konsekutive Lesarten sind bei Voranstellung ausgeschlossen. Variable in vorangestellten cosicché-Sätzen können aus dem zweiten Teilsatz heraus gebunden werden; phorische Ausdrücke im cosicchéSatz können koreferent mit Ausdrücken im zweiten Teilsatz sein:

(16a) Cosicché $\emptyset_{\mathrm{i}}$ possa aiutare a liberare l'altra metà della razza umana, [ogni donna $]_{\mathrm{i}}$ deve essere liberata.

damit $\emptyset_{\mathrm{i}}$ können-3sg-konj-präs helfen-inf zu befreien-inf die andere Hälfte der Menschheit, [jede Frau $]_{i}$ müssen-3sg-präs sein-inf befreien-partII

'Damit sie ${ }_{i}$ dabei helfen kann, die andere Hälfte der Menschheit zu befreien, muss [jede Frau] $]_{i}$ befreit werden.'

(16b) Cosicché $\emptyset_{\mathrm{i}}$ possano aiutare a liberare l'altra metà della razza umana, [le donne $]_{\mathrm{i}}$ devono essere liberate.

damit $\emptyset_{\mathrm{i}}$ können-3pl-konj-präs helfen-inf zu befreien-inf die andere Hälfte der Menschheit, [die Frauen] müssen-3pl-präs sein-inf befreien-partII

'Damit sie $_{i}$ dabei helfen können, die andere Hälfte der Menschheit zu befreien, müssen [die Frauen] $]_{i}$ befreit werden.' 
Finale cosicché-Sätze sind demnach eindeutig subordiniert und syntaktisch integriert; finales cosicché ist zweifelsfrei ein Subjunktor.

Cosicché scheint in seiner historischen Entwicklung also einen Schritt weiter gegangen zu sein als sodass. Konsekutive cosicché-Sätze sind desintegriert. Betrachtet man sie als adverbiale Adjunkte, so müssen sie im rechten Außenfeld des Matrixsatzes stehen und sind als Erweiterungen zur $\mathrm{CP}$ zu deuten. Nachgestellte finale cosicché-Sätze können dagegen als Erweiterungen zur IP gedeutet werden. Sie sind syntaktisch in den Matrixsatz integriert. Der Begriff des Postponierers könnte unter einer solchen Analyse dazu beitragen, das konsekutive cosicché von den prototypischen Subjunktoren wie auch von den Konjunktoren (deren Zweitkonnekt zum Erstkonnekt nicht in einer Adjunktbeziehung steht) abzugrenzen.

Auffällig ist, dass bei cosicché ebenso wie bei sodass die Entwicklung zum vollwertigen Subjunktor mit einem Bedeutungswandel von der konsekutiven zur finalen Lesart einhergeht. Dieser Übergang entspricht der generellen Bedeutungsentwicklung von Funktionswörtern, die (so gut wie) ausnahmslos von temporalen zu epistemischen bzw. deontischen Bedeutungen fortschreitet und (so gut wie) niemals umgekehrt (vgl. Traugott 1989; Sweetser 1990; Traugott/König 1991, S. 194ff.; Bybee/Perkins/Pagliuca 1994, S. 289; Keller 1995; Traugott/Dasher 2002, S. 34ff.). Konsekutivverknüpfungen betreffen typischerweise die satzsemantische Ebene der Sachverhalte; sie verknüpfen zeitliche Objekte (e-Objekte im Sinne von Blühdorn/Lohnstein 2012, S. 174f.). Finalverknüpfungen betreffen dagegen deontische Objekte. Diese gehören zur nächsthöheren Ebene der Satzsemantik (zur Ebene der m-Objekte im Sinne von Blühdorn/Lohnstein 2012, S. 176ff.). Die Voranstellbarkeit finaler cosicché-Sätze kann ebenso wie die Nicht-Voranstellbarkeit konsekutiver mit der Semantik in Zusammenhang gebracht werden. Ein Zweck ist eine beabsichtigte Wirkung. Die Absicht geht der Handlung voraus, die die Wirkung hervorbringen soll. Die Voranstellung des finalen cosicché-Satzes kann demnach ebenfalls als ikonisch gelten.

\subsection{Tanto più che}

Wie cosicché ist auch tanto più che durch die Verschiebung eines Adverbs (tanto più, 'umso mehr') aus dem Hauptsatz an die Spitze des nachgestellten Nebensatzes entstanden. Die Univerbierung mit che ist aber bisher ausgeblieben.

Stärker als cosicché-Sätze sind tanto più che-Sätze auf Nachstellung festgelegt (vgl. Lombardi Vallauri 2000, S. 82ff.). Sie geben eine Begründung für das im ersten Teilsatz Gesagte, und zwar eine zusätzliche Begründung, die über eine schon vorausgesetzte hinausgeht. Tanto più che bedeutet so viel wie 'auch weil', 'zumal'. Die Nicht-Voranstellbarkeit des tanto più che-Satzes kann semantisch bzw. pragmatisch erklärt werden - Zusätze folgen typischerweise nach:

(17) Non voglio entrare nei dettagli, tanto più che ho pochissimo tempo.

nicht wollen-1sg-präs gehen-inf in die Einzelheiten, zumal haben-1sg-präs sehr wenig Zeit 'Ich möchte nicht in die Einzelheiten gehen, zumal ich sehr wenig Zeit habe.'

Eine Variablenbindung in nachgestellte tanto più che-Sätze hinein ist nicht möglich:

(18) [Ogni studente $]_{\mathrm{i}}$ deve fare una richiesta, se/*tanto più che $\emptyset_{\mathrm{i}}$ è ambizioso

[jeder Student $]_{i}$ sollen-3sg-präs machen-inf einen Antrag, wenn/zumal $\emptyset_{i}$ sein-3sg-präs ehrgeizig

'[Jeder Student $]_{\mathrm{i}}$ soll einen Antrag stellen, wenn/* zumal er ${ }_{\mathrm{i}}$ ehrgeizig ist.' 
Aufgrund dieser Daten könnte tanto più che ebenso wie das konsekutive cosicché als Postponierer eingestuft werden. Allerdings ist die Voranstellung eines tanto più che-Satzes (zumindest in der Umgangssprache) nicht völlig ausgeschlossen. Anstelle von (17) wäre nach unserem Sprachgefühl auch (17a) denkbar, wenn der vorangestellte tanto più che-Satz in der prosodischen Phrasierung vom Restsatz abgetrennt wird:

(17a) Tanto più che ho pochissimo tempo, non voglio entrare nei dettagli.

auch weil haben-1sg-präs sehr wenig Zeit, nicht wollen-1sg-präs eintreten-inf in die Einzelheiten

'Gerade weil ich sehr wenig Zeit habe, will ich nicht in die Einzelheiten gehen.'

Diese Anordnung entspricht der natürlichen Abfolge von Ursache und Wirkung, ist also in anderer Weise ikonisch als (17) (zur ikonischen Konnektfolge in Kausalverknüpfungen vgl. Simone 1995, S. 166). In (19) ist der vorangestellte tanto più che-Satz durch einen Doppelpunkt vom darauffolgenden Teilsatz abgegrenzt. Im Gesprochenen würde dem wiederum eine besondere prosodische Gestaltung, z.B. eine Pause, ein Tonwechsel o. Ä. entsprechen:

(19) Tanto più che dici che la tua bimba ride molto: vedrai che un giorno ti stupirà con quella inconfondibile risata.

auch weil sagen-2sg-präs dass die deine Tochter lachen-3sg-präs viel: sehen-2sg-fut dass einen Tag dich erstaunen-3sg-fut mit jenem unverwechselbaren Lachen

'Gerade weil du sagst, dass deine Tochter viel lacht: Du wirst sehen, dass sie dich eines Tages mit ihrem unverwechselbaren Lachen erstaunen wird.'

(17a) und (19) sind so zu lesen, dass tanto più che Sprechakte (a-Objekte im Sinne von Blühdorn/Lohnstein 2012, S. 178f.) verknüpft. Sprechaktbezogene Nebensätze sind generell häufig syntaktisch desintegriert. Für (17a) und (19) würde das bedeuten, dass die tanto più che-Sätze im linken Außenfeld des Matrixsatzes (der LEA-Position im Sinne von Blühdorn 2012, S. 201) stehen, also linke CP-Adjunkte sind.

$\mathrm{Ob}$ vorangestellte tanto più che-Sätze auch syntaktisch integriert werden können, ist nach unserem Sprachgefühl fraglich. Variable, die in ihnen enthalten sind, können nicht aus dem übergeordneten Teilsatz heraus gebunden werden:

$(17 \mathrm{~b}) *$ Tanto più che $\emptyset_{\mathrm{i}}$ ha pochissimo tempo, nessuno vuole entrare nei dettagli.

zumal $\emptyset_{i}$ haben-3sg-präs sehr wenig Zeit, niemand ${ }_{i}$ wollen-3sg-präs gehen in die Einzelheiten

'Zumal er ${ }_{i}$ sehr wenig Zeit hat, will niemand in die Einzelheiten gehen.'

Tanto più che-Sätze, die nicht sprechaktbezogen, sondern im Sinne von Blühdorn/Lohnstein $(2012$, S. 188ff.) propositions- oder sachverhaltsbezogen sind und prosodisch in den Gesamtsatz integriert werden müssten, können nicht vorangestellt werden:

(20) *Tanto più che questo tipo di vino è impiantato ovunque in Grecia l'ipotesi dell'origine greca è fondata.

auch weil diese Weinsorte sein-3sg-präs anpflanzen-partII überall in Griechenland die Hypothese von der Herkunft griechischen ist begründet

'Gerade weil diese Weinsorte überall in Griechenland angepflanzt wird, ist die Vermutung ihrer griechischen Herkunft begründet.' 
Tanto più che-Sätze scheinen demnach weder bei Nachstellung noch bei Voranstellung syntaktisch integrierbar zu sein. Bei Nachstellung könnte tanto più che als Postponierer klassifiziert werden. Bei Voranstellung wäre eine solche Einordnung jedoch nicht möglich. Ebensowenig kann es dann als Subjunktor oder Konjunktor betrachtet werden. Tanto più che wäre somit einer Subkategorie der konjunktionalen Konnektoren zuzuordnen, die bei Pasch et al. (2003) nicht vorgesehen ist.

\section{$2.4 \quad$ Perché}

Sätze, die durch perché ('weil') eingeleitet sind, werden nach übereinstimmender Meinung der Grammatiker in aller Regel nachgestellt (vgl. Renzi/Salvi/Cardinaletti Bd. 2, 1991, S. 740; Lombardi Vallauri 2000, S. 64). Dennoch werden perché-Sätze durchweg als Nebensätze und perché selbst als Subjunktor betrachtet.

Im Korpus von Ravetto/Blühdorn (2011, S. 218ff.) ist der perché-Satz in 7 von 170 Belegen $(4 \%)$ vorangestellt. In der Literatur finden sich Hinweise auf vier Bedingungen, unter denen die Voranstellung möglich ist:

(i) wenn die Verknüpfung final gedeutet werden soll

(ii) wenn ein Korrelatadverb nachfolgt

(iii) wenn der perché-Satz intonatorisch hervorgehoben ist

(iv) wenn der perché-Satz durch Satzspaltung hervorgehoben ist

$\mathrm{Zu}$ (i): In Verknüpfungen wie (21), in denen der vorangestellte perché-Satz nicht als informationsstrukturell hervorgehoben gekennzeichnet ist, muss er final gelesen werden (vgl. Renzi/Salvi/Cardinaletti Bd. 2, 1991, S. 820). Ähnlich wie in finalen cosicché-Sätzen (s.o. Abschnitt 2.2) muss das Verb im Konjunktiv stehen; häufig kommen Modalverben wie potere ('können') vor:

(21) Perché lei possa affrontare al meglio il nuovo ambiente, è fondamentale il ruolo di mediatore che riesce a svolgere il marito.

damit sie können-3sg-konj-präs gegenübertreten-inf bestmöglich der neuen Umgebung, ist grundlegend die Rolle von Vermittler die schaffen-3sg-präs zu spielen-inf der Ehemann 'Damit sie der neuen Umgebung bestmöglich gegenübertreten kann, ist es grundlegend, dass es ihrem Mann gelingt, die Rolle eines Vermittlers zu spielen.'

Die Voranstellung eines kausalen perché-Satzes ohne informationsstrukturelle Hervorhebung wie in (22a) ist fragwürdig. Anstelle von perché wird dann poiché verwendet wie in (22b) (vgl. Ravetto/Blühdorn 2011):

(22a) ?Perché non avevo la mia macchina, sono rimasto a casa. weil nicht haben-1sg-perf das mein Auto, sein-1sg-präs bleiben-partII zu Hause 'Weil ich mein Auto nicht hatte, bin ich zu Hause geblieben.'

(22b) Poiché non avevo la mia macchina, sono rimasto a casa. da nicht haben-1sg-perf das mein Auto, sein-1sg-präs bleiben-partII zu Hause 'Da ich mein Auto nicht hatte, bin ich zu Hause geblieben.'

$\mathrm{Zu}$ (ii): In Verknüpfungen wie (23) wird der vorangestellte perché-Satz durch das Korrelatadverb perciò ('deswegen') wiederaufgenommen. Man könnte hier von einer „Linksversetzung" im Sinne von Altmann (1981) sprechen. Der perché-Satz ist informationsstrukturell hervorgehoben (vgl. Renzi/Salvi/Cardinaletti Bd. 2, 1991, S. 748f.). In solchen 
Fällen ist eine kausale Deutung der Verknüpfung möglich; eine finale ist aber nicht ausgeschlossen, wenn sie sinnvoll ist, wie in (24):

(23) Perché è straniero, perciò Alì non trova lavoro.

weil sein-3sg-präs Ausländer, deswegen Alì nicht finden-3sg-präs Job

'Weil er Ausländer ist, deswegen findet Alì keinen Job.'

(24) Perché tu possa orientarti al meglio in questa vita, per questo motivo ho aperto un blog sulla bellezza e la vita.

damit du können-2sg-konj-präs orientieren-inf dich bestmöglich in diesem Leben, für diesen Grund haben-1sg-präs einrichten-partII einen Blog über die Schönheit und das Leben 'Damit du dich so gut wie möglich in diesem Leben orientieren kannst, darum habe ich einen Blog über die Schönheit und das Leben eingerichtet.'

$\mathrm{Zu}$ (iii): In (25) ist der perché-Satz durch prosodische Mittel als Informationsfokus gekennzeichnet (vgl. Renzi/Salvi/Cardinaletti Bd. 2, 1991, S. 747; Lombardi Vallauri 2000, S. 66):

(25) A: Perché non scrive email? warum nicht schreiben-3sg-präs Email?

'Warum schreibt er/sie keine Emails?'

B: perché riesce solo a LEggere le sue email non ne scrive nessuna weil schaffen-3sg-präs nur zu LEsen die seine Emails, nicht davon schreiben-3sg-präs keine

'weil er/sie es nur schafft seine/ihre emails zu LEsen schreibt er/sie keine'

$\mathrm{Zu}$ (iv): In (26) ist der perché-Satz vorangestellter Fokus in einem Spaltsatz:

(26) è perché PIOve che non usciamo sein-3sg-präs weil regnen-3sg-präs dass nicht ausgehen-1pl-präs 'es ist weil es REGnet dass wir nicht ausgehen'

In der historisch älteren Variante der Kausalverknüpfung mit nachgestelltem Kausalsatz ist die Abfolge der Teilsätze antiikonisch. Aus semantischen Gründen kann es wünschenswert sein, durch Voranstellung eine ikonische Abfolge der Teilsätze herzustellen. Mit perché ist das in der Gegenwartssprache nur unter besonderen informationsstrukturellen Bedingungen möglich. Ansonsten erlauben nur finale perché-Verknüpfungen die Voranstellung des Nebensatzes.

Bindungsdaten deuten darauf hin, dass perché in allen Verwendungsweisen den von ihm eingeleiteten Teilsatz syntaktisch integrieren kann. Variable in nachgestellten kausalen perché-Sätzen wie in (27) und in vorangestellten finalen perché-Sätzen wie in (28) können aus dem anderen Teilsatz heraus gebunden werden:

(27) Nessuno $_{\mathrm{i}}$ è disoccupato perché $\emptyset_{\mathrm{i}}$ è giovane.

niemand $_{i}$ sein-3sg-präs arbeitslos weil $\emptyset_{i}$ sein-3sg-präs jung

'Niemand ${ }_{i}$ ist arbeitslos, weil er ${ }_{\mathrm{i}}$ jung ist.'

(28) Perché $\emptyset_{\mathrm{i}}$ possa affrontare al meglio il nuovo ambiente, [ogni donna $]_{\mathrm{i}}$ deve essere supportata dal marito.

damit $\emptyset_{\mathrm{i}}$ können-3sg-konj-präs gegenübertreten-inf bestmöglich der neuen Umgebung, [jede Frau] $]_{i}$ müssen-3sg-präs sein-inf unterstützen-partII vom Ehemann

'Damit sie $_{\mathrm{i}}$ der neuen Umgebung bestmöglich gegenübertreten kann, muss [jede Frau $]_{\mathrm{i}}$ von ihrem Mann unterstützt werden.' 
Auch bei vorangestellten kausalen perché-Sätzen mit informationsstruktureller Hervorhebung ist die Variablenbindung aus dem anderen Teilsatz heraus möglich, wie der folgende Originalbeleg zeigt:

(29) È perché $\emptyset_{\mathrm{i}}$ sfugge al mio controllo, che [ogni carezza $]_{\mathrm{i}}$ diventa un enigma per me. sein-3sg-präs weil $\emptyset_{\mathrm{i}}$ entgehen-3sg-präs zu meiner Kontrolle, dass [jedes Streicheln] werden-3sg-präs ein Geheimnis für mich

'Es ist, weil es außerhalb meiner Kontrolle steht, dass [jedes Streicheln $]_{i}$ für mich geheimnisvoll ist.'

Sprachhistorisch sind perché-Verknüpfungen anders entstanden als cosicché- und tanto più che-Verknüpfungen. Es handelt sich ursprünglich um nachgestellte Nominalsätze, die durch den Subordinationsanzeiger che eingeleitet waren und die kausale Präposition per ergänzten. Präposition und che wurden univerbiert und entwickelten sich in Richtung Subjunktor (vgl. Battisti/Alessio Bd. 4, 1954, S. 2846). Die beschränkte Voranstellbarkeit zeigt aber, dass das kausale perché noch nicht zu einem prototypischen Subjunktor geworden ist. Das finale perché ist dagegen in der Gegenwartssprache ein vollwertiger Subjunktor. Gleiches gilt für poiché, das aus einem nach rechts verschobenen Adverb (poi, lat. post) entstanden ist und gegenwartssprachlich keine Beschränkung hinsichtlich Voranstellbarkeit aufweist.

\section{$2.5 \quad$ Zwischenfazit}

Wenn man die Kategorie des Postponierers auf das Italienische übertragen möchte, könnte man ihr das konsekutive cosicché zuordnen. ${ }^{5}$ Tanto più che und das kausale perché zeigen ein anderes syntaktisches Verhalten. Tanto più che-Sätze können außer der REA- auch die LEA-Position einnehmen, bleiben aber syntaktisch stets desintegriert. Kausales perché kann den von ihm eingeleiteten Teilsatz syntaktisch integrieren, und die Voranstellung des perché-Satzes ist unter bestimmten Bedingungen möglich. Diese Konnektoren zeigen weder das Verhalten prototypischer Subjunktoren noch das von Postponierern.

Historisch sind cosicché, tanto più che und perché gleichermaßen an der Spitze nachgestellter Nebensätze aus der Verschmelzung von Adverbien bzw. einer Präposition mit dem Subordinationsanzeiger che entstanden. Ihre Entwicklung zu vollwertigen Subjunktoren ist gegenwartssprachlich noch im Gange (vgl. König/van der Auwera 1988, S. 107; Bybee/ Perkins/Pagliuca 1994, S. 14, 40; Cristofaro 1998, S. 61f., 77). Alle drei Konnektoren illustrieren einen Entwicklungsweg, der von Adverbkonnektoren bzw. adverbialen Präpositionalphrasen zu Subjunktoren führt. Mit prototypischen Konjunktoren wie $e$ zeigen sie nur wenige grammatische Gemeinsamkeiten.

\section{Postponierer im Deutschen}

Die deutschen Konnektoren, die Pasch et al. (2003, S. 418ff.) zu den Postponierern rechnen, zerfallen in zwei Gruppen, zwischen denen wichtige syntaktische Unterschiede bestehen:

(i) Relativadverbien wie weshalb, weswegen, wobei, wodurch, wohingegen, womit, wonach u.a.;

(ii) Ausdrücke mit konjunktionaler Charakteristik wie als dass, andernfalls, bloß/nur dass, sodass, umso mehr/weniger als, zumal.

Ebenso vermutlich das konsekutive sicché (vgl. Serianni 1989, S. 541, 586; Schwarze 1995, S. 743), das wir in diesem Aufsatz nicht untersucht haben. 
Ausdrücke der Gruppe (i) sind nach ihrem syntaktischen Verhalten nicht den konjunktionalen, sondern den adverbialen Konnektoren zuzurechnen. Ausdrücke der Gruppe (ii) zeigen ein ähnliches Verhalten wie Subjunktoren, jedoch mit der Beschränkung auf Nachstellung. Wir gehen nacheinander auf beide Gruppen ein.

\subsection{Relativadverbien}

Relativadverbien haben den Status von Phrasen. Einige von ihnen können durch Wortgruppen substituiert werden, etwa weshalb durch aus welchem Grunde. Morphologisch handelt es sich um $w$-Ausdrücke, die typischerweise parallele Verwendungen als Relativ- und Interrogativadverbien zulassen (vgl. Pasch et al. 2003, S. 9ff., 422ff.; Duden 2009, S. 577ff.):

(30) Hier hast du zwanzig Mark, womit die Sache wohl bereinigt sein dürfte. (Pasch et al. 2003, S. 730)

(31) Womit dürfte die Sache wohl bereinigt sein?

In (30) fungiert womit als adverbialer Einleiter eines satzbezogenen Relativsatzes (vgl. Blühdorn 2013, S. 213f.); dieser ist im Sinne von Holler (2005) weiterführend. In (31) fungiert womit als Interrogativadverb. In beiden Fällen hat es innerhalb des Satzes, den es einleitet, die Funktion eines Satzglieds, und zwar einer adverbialen Angabe. Nach der heute allgemein anerkannten syntaktischen Analyse nehmen $w$-Adverbien im Interrogativ- wie auch im Relativsatz das Vorfeld ein (vgl. etwa Sternefeld 2008/2009, S. 362ff.; Blühdorn 2012, S. 198; 2013, S. 195f., 213f.).

Relativadverbien bestehen aus einer pronominalen Komponente (z.B. wo-) und einer präpositionalen (z.B. -mit). Die präpositionale Komponente ist im syntaktischen Sinne ihr Kopf. Sie steht für eine semantische Relation. Die pronominale Komponente, die referentiell an den vorausgehenden Satz anknüpft, fungiert syntaktisch als Ergänzung der präpositionalen Komponente. Sie steht für das interne Argument der semantischen Relation. Der durch das Relativadverb eingeleitete Teilsatz steht für deren externes Argument (vgl. Pasch et al. 2003, S. 9f.; Blühdorn 2012, S. 198).

Pasch et al. (2003, S. 425) sehen satzverknüpfende Relativadverbien in „einem Übergangsbereich zwischen traditionell 'relativisch' genannter und traditionell 'konjunktional' genannter Subordination von Sätzen“. Aus welchen genauen Eigenschaften eine Ähnlichkeit von Relativadverbien mit Subjunktoren resultiert, lassen sie offen.

Viel deutlicher sind hier zunächst einmal die Unterschiede. So sind Relativadverbien im Gegensatz zu Konjunktionen keine syntaktischen Köpfe. Als Univerbierungen aus präpositionalem Kopf und pronominalem Komplement, können sie keine weiteren Ergänzungen fordern. Der durch sie eingeleitete Satz wird nicht von ihnen regiert. Das unterscheidet sie grundsätzlich von Konjunktionen.

Dennoch gibt es Hinweise darauf, dass einzelne Relativadverbien sich zukünftig zu Konjunktionen entwickeln könnten. So kann etwa wohingegen, anders als die übrigen Relativadverbien, nur relativ, also zur Satzverknüpfung, verwendet werden. Interrogative Verwendungen sind ausgeschlossen:

(32) Maria ist kerngesund, wohingegen Hans öfter Infekte hat. (Pasch et al. 2003, S. 730)

(33) *Wohingegen hat Hans öfter Infekte? 
Bei Internetrecherchen findet man vereinzelt Belege, in denen wohingegen zwei Sätze verknüpft, deren Finitum nicht am Ende steht, z.B. zwei Verbzweitsätze:

(34) Im World Wide Web kann man die Seiten nicht umbiegen, wohingegen man kann sich elektronisch ein Lesezeichen erstellen, ein sogenanntes „Bookmark“.

Sätze wie (34) können so gedeutet werden, dass wohingegen die Position eines Konjunktors einnimmt, die Nullstelle im Sinne von Blühdorn (2012, S. 201) und Blühdorn/Ravetto (2014, S. 18). Alternativ kann man annehmen, dass es im linken Außenfeld (der LEAPosition) des von ihm eingeleiteten Satzes steht - eine generelle Stellungsoption für Adverbkonnektoren. Für die zweite Annahme spricht seine syntaktische und prosodische Desintegration, die im Schrifttext oft durch einen Doppelpunkt oder Gedankenstrich nach wohingegen angezeigt wird. Unter dieser Analyse wäre es denkbar, dass wohingegen zugleich im rechten Außenfeld des ersten und im linken Außenfeld des zweiten Teilsatzes steht, also zu beiden Teilsätzen als CP-Adjunkt fungiert und dadurch eine lockere syntaktische Verknüpfung zwischen ihnen herstellt. Die Alternative wäre, zwischen dem ersten Teilsatz und dem wohingegen-Satz in Fällen wie (34) keine syntaktische, sondern nur eine semantische Verbindung anzunehmen. Dann wäre weder wohingegen allein noch der zweite Teilsatz als ganzer als Adjunkt zum ersten zu deuten.

Auch Belege, in denen wohingegen wie ein Subjunktor einen Verbletztsatz im Vorfeld eines Verbzweitsatzes einleitet, lassen sich finden:

(35) Zwischen einem Profi-Journalisten und einem Amateur-Journalisten gebe es folgende Unterschiede: Wohingegen der Profi eine Ausbildung genossen hat, meist bezahlt wird und sich an qualitative (professionelle) Standards hält, besitzt der Amateur keine Ausbildung, wird oft nicht bezahlt und unterliegt keiner qualitativen Prüfung.

Belege wie (35) entsprechen nicht dem Standard. Dass wohingegen die Fähigkeit annimmt, den von ihm eingeleiteten Teilsatz in den anderen zu integrieren, deutet sich aber auch darin an, dass eine Variable in einem nachgestellten wohingegen-Satz aus dem anderen Teilsatz heraus gebunden werden kann:

(36) [Jeder deutsche Beamte $]_{i}$ kommt montags zu spät zur Arbeit, wohingegen er freitags zu $_{\mathbf{i}}$ früh geht.

Beleg (36) scheint unproblematisch zu sein, wurde allerdings von uns selbst konstruiert. Originalbelege mit dieser Charakteristik haben wir nicht gefunden. Im Allgemeinen deuten wir nachgestellte, durch wohingegen eingeleitete Verbletztsätze als rechte Adjunkte zur CP des übergeordneten Satzes. Wohingegen-Sätze mit gebundener Variable betrachten wir als Adjunkte zu dessen IP.

Ganz ähnliche Beobachtungen kann man an wobei machen. In seiner konzessiven Variante kann es ebenfalls nicht als Interrogativadverb gebraucht werden. In (38) muss wobei temporal-situierend gelesen werden:

(37) Er lacht nie, wobei er seine Zähne wirklich nicht verstecken muss. (Pasch et al. 2003, S. 729)

(38) Wobei muss er seine Zähne wirklich nicht verstecken? 
Auch wobei wird gelegentlich zur Einleitung von Sätzen mit vorangestelltem Finitum verwendet. In solchen Verknüpfungen kann es wie wohingegen als Konjunktor oder als desintegriertes Adverb in der LEA-Position seines Zweitkonnekts gedeutet werden. Ein Beleg aus einem Internetforum:

(39) (...) gut, dass wir das Geld nicht haben - so haben wir auch solche Probleme nicht (...) Oder (...) mit einem Bentley Continental GT zum Aldi fahren - wobei - das hat schon wieder Stil (...)

Für einen wobei-Satz in Vorfeldstellung haben wir ebenfalls einen Beleg gefunden. Hier verhält sich wobei wie ein Subjunktor. Solche Sätze entsprechen aber nicht dem Standard:

(40) Wobei ich sagen muss, dass ich auch nicht so ganz Hochdeutsch rede, glaube ich, dass die richtige Aussprache die zweite ist.

In Belegen wie (41) und (42) kann das Pronomen im wobei-Satz möglicherweise als Variable gedeutet werden, die durch den Quantifikator jeder im ersten Teilsatz gebunden ist. Die Verfügbarkeit solcher Lesarten würde darauf hindeuten, dass wobei den Satz, den es einleitet, syntaktisch in einen übergeordneten Satz integrieren kann. In (41) ist die wobeiVerknüpfung bevorzugt temporal-situierend zu lesen, in (42) eher konzessiv:

(41) [Jeder Spieler $]_{i}$ deckt seine Plättchen auf, wobei $\mathbf{e r}_{\mathrm{i}}$ das letzte zur Markierung quer legt.

(42) Teilnehmen kann jeder ${ }_{i}$, wobei er ${ }_{i}$ versuchen sollte, sich an die Regeln zu halten.

Durch Relativadverbien eingeleitete Teilsätze werden typischerweise nachgestellt. Sätze, deren Vorfeld mit einem $w$-Adverb besetzt ist, kommen aber durchaus auch vorangestellt vor, insbesondere mit Sprechaktbezug. Sie nehmen dann das linke Außenfeld des Gesamtsatzes ein; das $w$-Adverb scheint zugleich relative und interrogative Eigenschaften zu haben:

(43) (...) wir sind da nicht so fleißig was den Adventskalender angeht, und Emily bekommt einen von Playmobil (...). Weshalb ich das sage: Es gibt von Playmobil auch kleine einzelne Figürchen, die sich dafür bestimmt eignen würden.

In der Zusammenschau zeigen die Daten, dass die Einordnung der $w$-Adverbien als Postponierer problematisch ist. Durch $w$-Adverbien eingeleitete Teilsätze sind nicht absolut auf Nachstellung festgelegt. Die meisten $w$-Adverbien zeigen keine konjunktionsähnlichen Eigenschaften, sondern verhalten sich wie typische Adverbien. Nur bei wohingegen und wobei liegen Hinweise auf konjunktionsähnliches Verhalten vor. Es ist nicht ausgeschlossen, dass sie im Begriff sind, sich zu Subjunktoren zu entwickeln. Dann durchlaufen sie dabei aber einen anderen Weg als Ausdrücke wie sodass oder zumal.

\subsection{Ausdrücke mit konjunktionaler Charakteristik}

Im Gegensatz zu den meisten Ausdrücken der Gruppe (i) sind diejenigen der Gruppe (ii) als syntaktische Köpfe aufzufassen. In der Linearstruktur (vgl. Wöllstein 2010, S. 22ff., 32ff.) nehmen sie insgesamt oder zumindest ein Teil von ihnen die linke Verbposition des von ihnen eingeleiteten Teilsatzes ein. Der Rest dieses Teilsatzes ist ihr syntaktisches Komplement, d.h. ihr internes Konnekt. Dieses stellen sie ihrem externen Konnekt nach (s.o. Kap. 1; vgl. Blühdorn 2012, S. 199). 
Zumal und andernfalls sind von Haus aus Adverbien, die in bestimmten Kontexten als Konjunktionen reanalysiert werden. Zumal stammt aus dem mittelhochdeutschen Adverb ze māle ('zugleich', 'gleichzeitig'), das später die Funktion einer Fokuspartikel mit der Bedeutung 'vor allem', 'besonders' annahm. Seit dem 17. Jahrhundert tritt sie häufig vor adverbiale Nebensätze, insbesondere vor solche mit da oder wenn. Durch den Wegfall des Subjunktors rückt zumal selbst in dessen Position auf (vgl. Grimm/Grimm 1984, Bd. 32, S. 531ff.; Paul 1992, S. 1087; Kluge 2011, S. 1017):

(44) Die Eltern sind nicht mehr jung und bald zu schwach, um dem Sohn Grenzen zu setzen, zumal (da) er starke Verhaltensauffälligkeiten entwickelt.

Wegfall des Subjunktors und Reanalyse von zumal erfolgen ausschließlich in der Nachstellung (vgl. Lehmann 1988, S. 188). Bei Voranstellung des Nebensatzes wird der Subjunktor nicht weggelassen; zumal bleibt Fokuspartikel (vgl. Pasch 2000, S. 30):

(45) Zumal da nur fünf von ihnen dabei sein konnten, hatte die Plattdeutsch-AG der HansClausen-Schule mit ihren Darbietungen mehr Mühe.

Andernfalls wird in der Gegenwartssprache hauptsächlich als volles Adverb verwendet wie in (46). Es kann aber auch, vor allem im Verwaltungsstil, als Einleiter eines Verbletztsatzes dienen wie in (47):

(46) Radfahrer müssen einen vorhandenen Radweg nutzen - andernfalls sieht es bei einem Unfall mit Schadensersatzansprüchen schlecht aus!

(47) Die Ablehnung eines schriftlichen Auftrages haben wir binnen 14 Tagen nach dessen Einlangen schriftlich zu erklären, andernfalls er mit dem Datum der Auftragserteilung als angenommen gilt.

Die Voranstellung eines mit andernfalls eingeleiteten Nebensatzes ist ausgeschlossen. Auch eine Variablenbindung in einen andernfalls-Satz hinein scheint unmöglich zu sein. So scheidet etwa für (48) eine Lesart des Pronomens er als durch den Quantifikator kein gebundene Variable aus:

(48) [Kein Arbeitsloser $]_{i}$ darf es versäumen, sich regelmäßig zu melden, andernfalls er ${ }_{i}$ die Unterstützung verliert.

Er fungiert hier analog zu [jemand, der arbeitslos ist, $]_{i}$ darf es nicht versäumen, ..., andernfalls er $_{1} . .$. als referentiell wiederaufnehmende Proform, die nicht auf ein syntaktisches Unterordnungsverhältnis angewiesen ist.

Die Umkategorisierung von andernfalls zur Konjunktion scheint dadurch möglich geworden zu sein, dass in Zweitkonnekten, in denen es das Vorfeld einnahm, wie in (46), auf die Voranstellung des Finitums verzichtet wurde. Ein ähnlicher Fall ist gleichwohl, das in der Entwicklung zum Subjunktor weiter gegangen ist und den von ihm eingeleiteten Teilsatz auch voranstellen kann:

(49) Gleichwohl er Engländer ist, hat Miller ein fabelhaftes Ohr für Dialekte.

Im Deutschen wie in anderen Sprachen entwickeln sich Subjunktoren generell oft aus Adverbkonnektoren (vgl. Kortmann 1996, S. 27, 108ff.). Viele Subjunktoren des Deutschen stammen von $d$-Pronominaladverbien ab, z.B. indem, nachdem, seitdem, trotzdem u.a. (vgl. Kortmann 1998, S. 488). In ihrer Form erkennt man noch die präpositionale und die pronominale Komponente. Ursprünglich waren sie mit dem Komplementierer dass zusammengesetzt. Ihre Reanalyse als Subjunktoren ging mit dem Wegfall des Komple- 
mentierers einher (vgl. Behaghel 1932, S. 263). Auch adverbiale Antezedentien der Vergleichspartikeln wie bzw. als, etwa solange, sobald, sooft oder sofern, haben sich unter Wegfall der Vergleichspartikel zu Subjunktoren entwickelt. Überall ist die Umkategorisierung an der Spitze des Zweitkonnekts erfolgt.

Sodass ist ebenfalls aus einem Adverb (dem deiktischen so) und dem Komplementierer dass entstanden. So stand ursprünglich im Mittelfeld des Erstkonnekts, wurde nach rechts an den Anfang des Zweitkonnekts verschoben und dort mit dem Komplementierer dass univerbiert (vgl. Konerding 2002, S. 124ff.). Hier ist der Komplementierer nicht ausgefallen, sondern die Eigenschaft als syntaktischer Kopf ist von ihm auf die univerbierte Form übergegangen. Dass sodass-Sätze bisher keine Voranstellung entwickelt haben, wird von Behaghel (1932, S. 263), Zifonun/Hoffmann/Strecker (1997, S. 2311) und anderen Autoren mit semantischer Ikonizität erklärt.

Als dass (vgl. Duden 2009, S. 372, 522, 620, 632) ist im Gegensatz zu sodass nicht univerbiert. Es enthält kein Adverb, sondern besteht aus der Vergleichspartikel als und dem Komplementierer dass. Semantisch sind als dass-Sätze teils als Konsekutiv-, teils als Vergleichssätze zu charakterisieren. Nach Behaghel (1932, S. 264f.) gehören auch sie zu den Nebensätzen, die aus semantischen Gründen keine Voranstellung entwickelt haben:

(50) Das Problem ist zu komplex, als dass wir es im Handumdrehen lösen könnten. (Konsekutivverknüpfung)

(51) Mancher ertrinkt lieber, als dass er um Hilfe ruft. (Vergleichsverknüpfung)

Während die Abfolge der Teilsätze in Konsekutivverknüpfungen wie (50) ikonisch ist, ist in Vergleichsverknüpfungen wie (51) nicht durchsichtig, warum das mit als angeschlossene Vergleichsobjekt dem Verglichenen nachgestellt werden muss. In Vergleichen mit wie gilt diese Beschränkung nicht:

(52) Es schallt aus dem Wald, wie man hineinruft.

(52a) Wie man in den Wald ruft, (so) schallt es heraus.

Das konsekutive als dass scheint stärker lexikalisiert zu sein als das vergleichende. Im Standard verlangt es den Konjunktiv in dem von ihm eingeleiteten Teilsatz (vgl. Duden 2009, S. 522). Weder als noch dass zeigen allein eine solche Forderung. Das spricht dafür, das konsekutive als dass als lexikalisierte Konjunktion mit eigenen grammatischen Eigenschaften aufzufassen. Subordination und die Beschränkung auf Nachstellung kennzeichnen es als Postponierer.

Dagegen verhält sich als in vergleichenden als dass-Verknüpfungen wie die gewöhnliche Vergleichspartikel. Es folgt auf den Komparativ und auf einige wenige lexikalische Antezedentien wie anders und insofern und kann nicht nur dass-Sätzen, sondern Vergleichsausdrücken unterschiedlicher grammatischer Form vorangestellt werden, etwa $z u$-Infinitiven wie in (51a), Verbzweitsätzen wie in (53), Nominalgruppen wie in (54), Adverbialia wie in (55) und anderen:

(51a) Mancher ertrinkt lieber als um Hilfe zu rufen.

(53) Mancher ermüdet schneller als er arbeitet.

(54) Einer ermüdet schneller als der andere.

(55) Sie ermüden heute schneller als gestern. 
Diese Beobachtungen sprechen dafür, das vergleichende als dass nicht als lexikalische Einheit zu betrachten. Die Vergleichspartikel als ist ebenfalls ein Konnektor (vgl. Blühdorn 2008; 2013, S. 202f.) und auf Nachstellung des von ihr eingeleiteten Konnekts festgelegt, zeigt aber ein anderes syntaktisches Verhalten als die Postponierer.

Die Möglichkeit der Variablenbindung in Sätzen wie (51) deutet darauf hin, dass vergleichende als-Verknüpfungen syntaktisch integriert sind. Auch in konsekutiven als dass-Verknüpfungen wie (56) scheint die Variablenbindung nicht ausgeschlossen zu sein. Demnach hätte das konsekutive als dass wie sodass auf dem Entwicklungsweg zum Subjunktor schon eine Strecke zurückgelegt:

(51) Mancher $_{i}$ ertrinkt lieber, als dass er ${ }_{i}$ um Hilfe ruft.

(56) Niemand ${ }_{i}$ war zu höflich, als dass er $_{i}$ sich beschwert hätte.

Bloß dass und nur dass bestehen aus Fokuspartikeln und dem Komplementierer dass. Im Deutschen werden Fokuspartikeln ihrer Bezugskonstituente typischerweise unmittelbar vorangestellt (vgl. Dimroth 2004, S. 57ff.). Bei bloß dass und nur dass ist die Bezugskonstituente der dass-Satz. Da keine Univerbierung stattgefunden hat, ist unklar, ob blo $\beta$ dass und nur dass als komplexe Konjunktionen aufzufassen sind. Alternativ können blo $\beta$ und nur als selbständige Einheiten analysiert werden, die ihrerseits Konnektorfunktion haben (vgl. Blühdorn 2008). Dafür spricht, dass sie nicht nur dass-Sätzen wie in (57), sondern auch Ausdrücken anderer grammatischer Form, z.B. Verbzweitsätzen wie in (58), vorangestellt werden können. Ferner können sie, wie auch andere Fokuspartikeln, als Satzglieder fungieren und im Vorfeld von Verbzweitsätzen stehen wie in (59):

(57) Die Antifa will mehr Sozialismus. Bloß dass niemand definiert, was Sozialismus überhaupt sein kann.

(58) Professor $Z$ ist fachlich der größere Experte. Bloß, niemand weiß es.

(59) Auch in der Ukraine gibt es eine Piraten-Partei. Bloß hat sie keinen Schimmer von politischer Ahnung.

$B l o \beta$ wäre in allen diesen Beispielen annähernd bedeutungsgleich durch nur ersetzbar. Es kündigt eine Einschränkung zu dem im Erstkonnekt Gesagten an. Aus dieser semantischpragmatischen Funktion erklärt sich die Nicht-Voranstellbarkeit des $b l o \beta$ - bzw. nur-Satzes. In der Schreibung werden $b l o \beta$ - und nur-Sätze typischerweise durch ein auf Desintegration hinweisendes Interpunktionszeichen (Punkt, Gedankenstrich o.Ä.) vom Erstkonnekt getrennt. Soweit sie überhaupt als Teile von Satzgefügen verstanden werden können, kommen sie wohl allenfalls als CP-Adjunkte in Frage. Variablenbindung in bloß- und $n u r$-Sätze hinein scheint ausgeschlossen zu sein:

(60) *Niemand $_{i}$ wollte auf Ottos Party fehlen, bloß dass er $_{\mathbf{i}}$ kein Geschenk mitbringen wollte.

Auch umso mehr/weniger als sind nicht univerbiert. Sie bestehen aus einer adverbialen Vergleichsphrase mit Komparativ (z.B. umso mehr) und anschließendem als. Die Vergleichsphrase kann, je nach Kontext, auch mit anderen Komparativen gebildet werden, etwa umso eher, umso stärker, umso lieber oder umso besser. Anstelle von als können auch Ausdrücke wie da, wo, wenn oder weil stehen. Ob der Anschlussausdruck syntaktisch als Vergleichspartikel, Subjunktor oder Relativadverb zu deuten ist, kann von Kontext zu Kontext variieren und bleibt häufig unklar. 
Die vielfältigen Ersetzungsmöglichkeiten lassen Zweifel daran aufkommen, dass es sich bei umso mehr/weniger als um Lexikalisierungen handelt. Die Vergleichsphrase kann als adverbiale Angabe im Mittelfeld des Matrixsatzes wie in (61a), am Ende des Matrixsatzes wie in (61b) oder als Fokuspartikel an der Spitze des anschließenden Vergleichspartikel-, Subjunktor- oder Relativsatzes stehen wie in (61c):

(61a) Die Festival-Besucher haben sich umso mehr gefreut, als/da die Sonne herauskam.

(61b) Die Festival-Besucher freuten sich umso mehr, als/da die Sonne herauskam.

(61c) Die Festival-Besucher freuten sich, umso mehr als/da die Sonne herauskam.

Steht die Vergleichsphrase im Mittelfeld des Erstkonnekts, so sind oft Lesarten naheliegend, in denen das Zweitkonnekt die gegebene Sachverhaltsbeschreibung modifiziert (temporale Verknüpfung im Sinne von Blühdorn 2012, S. 194ff. und Blühdorn/Lohnstein 2012, S. 189ff.). Steht die Vergleichsphrase dagegen an der Spitze des Zweitkonnekts, so treten Lesarten in den Vordergrund, in denen die Teilsätze auf der Sprechaktebene verknüpft sind. Solche Lesarten scheinen eine Reanalyse der Vergleichsphrase und ihres Anschlussausdrucks als Mehrwortkonnektor zu begünstigen. Gegenwartssprachlich kann man hier aber noch kaum von Lexikalisierung sprechen; es liegen noch weitgehend freie syntaktische Fügungen vor.

Sätze wie (62) deuten darauf hin, dass eine Variablenbindung in sprechaktverknüpfte umso-Komparativ-Sätze hinein ausgeschlossen ist:

(62) Niemand ${ }_{i}$ wollte auf Ottos Party gehen, weil/?? umso weniger als $\mathrm{er}_{i}$ etwas zu verschenken hatte.

Während das Pronomen er in der Verknüpfung mit weil problemlos als durch niemand gebundene Variable gelesen werden kann, kommt in der Verknüpfung mit umso weniger als allenfalls seine Deutung als referentiell wiederaufnehmende Proform in Frage. Die Konnekte einer umso-Komparativ-Verknüpfung scheinen syntaktisch demnach desintegriert zu bleiben.

\subsection{Zusammenfassung}

Die Postponierer im Sinne von Pasch et al. (2003, S. 418ff.) bilden eine in mehrfacher Hinsicht uneinheitliche Gruppe. Die w-Adverbien unter ihnen zeigen bis auf wohingegen und wobei keine Eigenschaften von Konjunktionen. Unter den Ausdrücken mit konjunktionaler Charakteristik sind bloß/nur dass, umso mehr/weniger als und das vergleichende als dass nicht lexikalisiert. Nur zumal, sodass, das konsekutive als dass und das konjunktionale andernfalls zeigen ein syntaktisches Verhalten, das ihre Einordnung als Postponierer im Sinne der Definitionskriterien rechtfertigt.

\section{Ausblick und Schlussfolgerungen}

In den vorherigen Abschnitten wurden Pfade der diachronen Entwicklung angesprochen, die bei Konnektoren beobachtet werden können: 


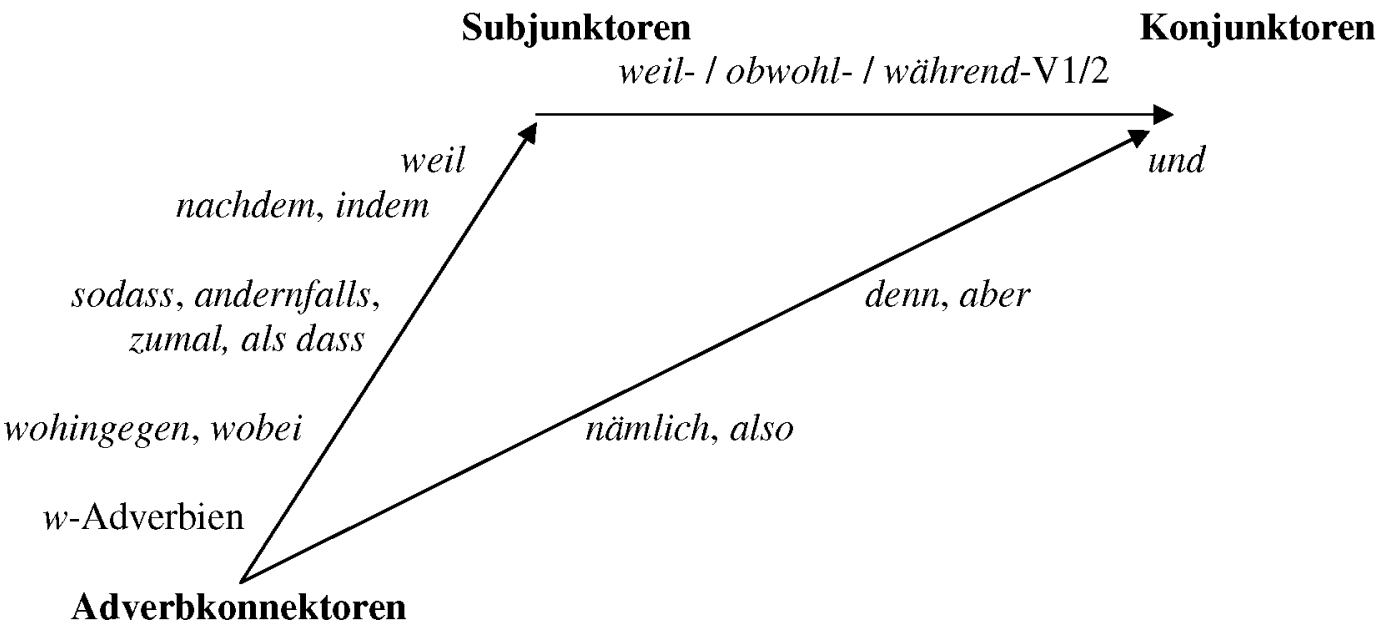

Abb. 3: Pfade der diachronen Entwicklung bei Konnektoren des Deutschen

Das Schema zeigt drei Entwicklungspfade, von denen einer im Mittelpunkt dieser Arbeit stand: Adverbkonnektoren entwickeln sich zu Subjunktoren. Die deutschen $w$-Adverbien als Einleiter von Interrogativ- und Relativsätzen bewahren im Wesentlichen ihren Adverbcharakter. Lediglich wohingegen und wobei zeigen in Ansätzen Eigenschaften von Subjunktoren. Sodass, andernfalls und zumal sowie das konsekutive als dass haben mehrere, aber nicht alle Subjunktoreigenschaften angenommen. Das gilt auch für das konsekutive cosicché im Italienischen. Tanto più che zeigt demgegenüber noch deutlich weniger Ähnlichkeit mit einem Subjunktor. Das kausale perché zeigt mehr, das finale perché sämtliche Subjunktoreigenschaften. Die deutschen Pronominaladverbien nachdem, indem usw. kommen parallel als vollwertige Subjunktoren vor. Auch Subjunktoren wie weil (aus ahd dia wīla sō; vgl. Kluge 2011, S. 979), deren Herkunft gegenwartssprachlich nicht mehr transparent ist, gehen auf adverbiale Ausdrücke zurück.

Zwei Entwicklungspfade, die nicht im Mittelpunkt der Arbeit standen, führen von Adverbien bzw. Subjunktoren zu Konjunktoren. Deutsch nämlich und also sind Adverbien, die vereinzelt in Konjunktorfunktion vorkommen (vgl. Blühdorn 2012, S. 201); denn und aber haben parallele Verwendungen als Adverbien und Konjunktoren; und ist gegenwartssprachlich ein reiner Konjunktor, stammt aber wahrscheinlich aus einer adverbialen Wurzel (idg. *hanti 'auf der Vorderseite'; vgl. Paul 1992, S. 938; Kluge 2011, S. 941). Für die Entwicklung von Subjunktoren zu Konjunktoren sind weil, obwohl und während mit Verberst- bzw. Verbzweitsatzergänzung mögliche Beispiele (vgl. Keller 1995; Uhmann 1998, S. 95f; Wegener 1999, S. 9f.; Duden 2009, S. 1218ff.; Freywald 2010).

Wenn Pasch et al. (2003, S. 352) die deutschen Postponierer als „Übergangsklasse zwischen Subjunktoren und Konjunktoren" charakterisieren, so ist dies eine rein klassifikatorische Kennzeichnung, die dem sprachgeschichtlichen Befund nicht entspricht und auch der gegenwartssprachlichen Grammatik dieser Untergruppe der konjunktionalen Konnektoren letztlich nicht gerecht wird. Unter beiden Blickwinkeln sind Postponierer auf der Skala zwischen Adverbkonnektoren und Subjunktoren einzuordnen. Mit Konjunktoren zeigen sie keine echten Ähnlichkeiten - mit der Ausnahme von wohingegen und wobei, die gelegentlich auch Verberst- oder Verbzweitsätze einleiten. 
Unsere Schlussfolgerungen werden durch Sprachvergleichsdaten bestärkt. In diesem Aufsatz wurden Konnektoren des Italienischen herangezogen, die in ihrer historischen Entwicklung und ihren gegenwartssprachlichen Eigenschaften den deutschen Postponierern ähneln. Im Italienischen wird noch deutlicher, wie klein und uneinheitlich diese Gruppe ist. Auch im Deutschen bleiben nach einer genauen syntaktischen Analyse nur vier Konnektoren übrig, die die Definitionskriterien für Postponierer erfüllen. In seiner Entstehungsgeschichte und seinem genauen grammatischen Verhalten unterscheidet sich jeder von ihnen noch einmal von den übrigen.

Vor diesem Hintergrund stellt sich nicht nur für die Grammatik des Italienischen und für den Sprachvergleich, sondern auch für die Grammatik des Deutschen die Frage nach der faktischen Nützlichkeit des Postponiererbegriffs. Wahrscheinlich wird man den betreffenden Konnektoren letztlich besser gerecht, wenn man sie im Sinne von Pasch et al. (2003, S. 584ff.) als grammatische Einzelgänger einstuft und auf die Kategorie der Postponierer verzichtet.

\section{Literatur}

Altmann, Hans (1981): Formen der 'Herausstellung' im Deutschen. Rechtsversetzung, Linksversetzung, Freies Thema und verwandte Konstruktionen. Tübingen: Niemeyer. (= Linguistische Arbeiten 106).

Battisti, Carlo/Alessio, Giovanni (1950-1957): Dizionario etimologico italiano. 5 Bde. Firenze: Barbera.

Behaghel, Otto (1932): Deutsche Syntax. Eine geschichtliche Darstellung. Bd. 4: Wortstellung. Periodenbau. Heidelberg: Winter. (= Germanische Bibliothek 10).

Blühdorn, Hardarik (2008): Syntax und Semantik der Konnektoren. Ein Überblick. Online unter: wwwl. ids-mannheim.de/fileadmin/gra/texte/blu_ueberblick.pdf (Stand: 15.12.2015).

Blühdorn, Hardarik (2012): Verknüpfungseigenschaften von Satzkonnektoren im Deutschen. Am Beispiel der Kausal- und Konsekutivkonnektoren. In: Deutsche Sprache 40, S. 193-220.

Blühdorn, Hardarik (2013): Syntaktische Nebensatzklassen im Deutschen. In: Deutsche Sprache 41, S. 193-219.

Blühdorn, Hardarik/Lohnstein, Horst (2012): Verumfokus im Deutschen: Versuch einer Synthese. In: Lohnstein, Horst/Blühdorn, Hardarik (Hg.): Wahrheit - Fokus - Negation. Hamburg: Buske. S. 171-262. (= Linguistische Berichte. Sonderheft 18).

Blühdorn, Hardarik/Ravetto, Miriam (2014): Satzstruktur und adverbiale Subordination. Eine Studie zum Deutschen und zum Italienischen. In: Linguistik online 67. Online unter: https://bop.unibe.ch/linguistikonline/article/view/1598 (Stand: 15.12.2015).

Boettcher, Wolfgang/Sitta, Horst (1972): Deutsche Grammatik. Teil 3: Zusammengesetzter Satz und äquivalente Strukturen. Frankfurt a.M.: Athenäum. (= Studienbücher zur Linguistik und Literaturwissenschaft 4).

Breindl, Eva/Volodina, Anna/Waßner, Ulrich Hermann (2014): Handbuch der deutschen Konnektoren 2: Semantik der deutschen Satzverknüpfer. Berlin/Boston: De Gruyter. (= Schriften des Instituts für Deutsche Sprache 13).

Bybee, Joan/Perkins, Revere/Pagliuca, William (1994): The evolution of grammar. Tense, aspect, and modality in the languages of the world. Chicago: University of Chicago Press.

Christ, Rüdiger (2014): Zur Satzfügung im Deutschen. Vornehmlich am Beispiel adversativer Satzgefüge. Tübingen: Stauffenburg. (= Studien zur deutschen Grammatik 86).

Cristofaro, Sonia (1998): Grammaticalization and clause linkage strategies. In: Giacalone Ramat, Anna/ Hopper, Paul J. (Hg.): The limits of grammaticalization. Amsterdam: Benjamins. S. 59-88. (= Typological Studies in Language 37). 
Cristofaro, Sonia (2003): Subordination. Oxford: Oxford University Press.

Dimroth, Christine (2004): Fokuspartikeln und Informationsgliederung im Deutschen. Tübingen: Stauffenburg. (= Studien zur deutschen Grammatik 69).

Duden (2009): Die Grammatik. 8., überarb. Aufl. Mannheim: Dudenverlag. (= Der Duden 4).

Engel, Ulrich (1988): Deutsche Grammatik. Heidelberg: Groos.

Freywald, Ulrike (2010): Obwohl vielleicht war es ganz anders. Vorüberlegungen zum Alter der Verbzweitstellung nach subordinierenden Konjunktionen. In: Ziegler, Arne (Hg.): Historische Textgrammatik und historische Syntax des Deutschen. Traditionen, Innovationen, Perspektiven. Berlin/New York: De Gruyter. S. 55-84.

Grimm, Jakob/Grimm, Wilhelm (1984): Deutsches Wörterbuch. 33 Bde. München: Deutscher Taschenbuch Verlag.

Haspelmath, Martin (1995): The converb as a cross-linguistically valid category. In: Haspelmath, Martin/ König, Ekkehard (Hg.): Converbs in cross-linguistic perspective. Structure and meaning of adverbial verb forms, adverbial participles, gerunds. Berlin/New York: De Gruyter. S. 1-55. (= Empirical Approaches to Language Typology 13).

Helbig, Gerhard/Buscha, Joachim (1972): Deutsche Grammatik. Ein Handbuch für den Ausländerunterricht. Leipzig: Verlag Enzyklopädie.

Holler, Anke (2005): Weiterführende Relativsätze. Empirische und theoretische Aspekte. Berlin: Akademie-Verlag.

Holler, Anke (2007): Uniform oder different? Zum syntaktischen Status nicht-restriktiver Relativsätze. In: Deutsche Sprache 35, S. 250-270.

Holler, Anke (2008): German dependent clauses from a constraint-based perspective. In: Fabricius-Hansen, Cathrine/Ramm, Wiebke (Hg.): 'Subordination' versus 'coordination' in sentence and text. A cross-linguistic perspective. Amsterdam/Philadelphia: Benjamins. S. 187-216. (= Studies in Language 98).

Johannessen, Janne Bondi (1998): Coordination. New York: Oxford University Press.

Keller, Rudi (1995): The epistemic weil. In: Stein, Dieter/Wright, Susan (Hg.): Subjectivity and subjectivization. Linguistic perspectives. Cambridge: Cambridge University Press. S. 16-30.

Kluge (2011): Etymologisches Wörterbuch der deutschen Sprache. 25., durchges. u. erw. Aufl., bearbeitet von Elmar Seebold. Berlin/Boston: De Gruyter.

Konerding, Klaus-Peter (2002): Konsekutivität als grammatisches und diskurspragmatisches Phänomen. Tübingen: Stauffenburg. (= Studien zur deutschen Grammatik 65)

König, Ekkehard/van der Auwera, Johan (1988): Clause integration in German and Dutch conditionals, concessive conditionals, and concessives. In: Haiman, John/Thompson, Sandra (Hg.): Clause combining in grammar and discourse. Amsterdam/Philadelphia: Benjamins. S. 101-133. (= Typological Studies in Language 18).

König, Ekkehard/van der Auwera, Johan (1990): Adverbial participles, gerunds and absolute constructions in the languages of Europe. In: Bechert, Johannes/Bernini, Giuliano/Buridant, Claude (Hg.): Toward a typology of European languages. Berlin/New York: De Gruyter. S. 337-355. (= Empirical Approaches to Language Typology 8).

Kortmann, Bernd (1996): Adverbial subordination. A typology and history of adverbial subordinators based on European languages. Berlin: De Gruyter. (= Empirical Approaches to Language Typology 18).

Kortmann, Bernd (1998): Adverbial subordinators in the languages of Europe. In: van der Auwera, Johan/ Baoill, Dónall P. Ó. (Hg.): Adverbial constructions in the languages of Europe. Berlin: De Gruyter. S. 457-561. (= Empirical Approaches to Language Typology 3). 
Lehmann, Christian (1988): Towards a typology of clause linkage. In: Haiman, John/Thompson, Sandra (Hg.): Clause combining in grammar and discourse. Amsterdam/Philadelphia: Benjamins. S. 181-225. (= Typological Studies in Language 18).

Lohnstein, Horst (2014): Artenvielfalt in freier Wildbahn - Generative Grammatik. In: Hagemann, Jörg/ Staffeldt, Sven (Hg.): Syntaxtheorien. Analysen im Vergleich. Tübingen: Stauffenburg. S. 165-185. (= Stauffenburg-Einführungen 28).

Lombardi Vallauri, Edoardo (2000): Grammatica funzionale delle avverbiali italiane. Roma: Carocci. (= Biblioteca di Testi e Studi 134).

Pasch, Renate (2000): Vorschlag für eine Neuordnung der „subordinierenden“ Konjunktionen des Deutschen. In: Lefèvre, Michel (Hg.): Subordination in Syntax, Semantik und Textlinguistik. Tübingen: Stauffenburg. S. 23-32. (= Eurogermanistik 15).

Pasch, Renate/Brauße, Ursula/Breindl, Eva/Waßner, Ulrich Hermann (2003): Handbuch der deutschen Konnektoren. Linguistische Grundlagen der Beschreibung und syntaktische Merkmale der deutschen Satzverknüpfer. Berlin/New York: De Gruyter. (= Schriften des Instituts für Deutsche Sprache 9).

Paul, Hermann (1992): Deutsches Wörterbuch. 9., vollst. neu bearb. Aufl. von Helmut Henne und Georg Objartel unter Mitarbeit von Heidrun Kämper-Jensen. Tübingen: Niemeyer.

Pittner, Karin (1999): Adverbiale im Deutschen. Untersuchungen zu ihrer Stellung und Interpretation. Tübingen: Stauffenburg. (= Studien zur deutschen Grammatik 60).

Previtera, Luisa (1996): I costrutti causali. In: Prandi, Michele (Hg.): La subordinazione non completiva. Un frammento di grammatica filosofica, S. 29-46. (= Studi italiani di linguistica teorica e applicata 25,1).

Quirk, Randolph et al. (1985): A comprehensive grammar of the English language. London: Longman.

Ravetto, Miriam/Blühdorn, Hardarik (2011): Die Kausalkonjunktionen denn, weil, da im Deutschen und perché, poiché, siccome im Italienischen. In: Ferraresi, Gisella (Hg.): Konnektoren im Deutschen und im Sprachvergleich. Beschreibung und grammatische Analyse. Tübingen: Narr. S. 207-250. (= Studien zur Deutschen Sprache 53).

Ravetto, Miriam/Blühdorn, Hardarik (in Vorb.): Satzverknüpfungen mit Konjunktionen und Adverbkonnektoren Deutsch-Italienisch: Syntax und Semantik. Erscheint in: OPAL. Online publizierte Arbeiten zur Linguistik. Mannheim: Institut für Deutsche Sprache.

Renzi, Lorenzo/Salvi, Giampaolo/Cardinaletti, Anna (1988-1995): Grande grammatica italiana di consultazione. 3 Bde. Bologna: Il Mulino.

Salvi, Giampaolo/Vanelli, Laura (2004): Nuova grammatica italiana. Bologna: Il Mulino.

Schwarze, Christoph (1995): Grammatik der italienischen Sprache. 2., verb. Aufl. Tübingen: Niemeyer.

Serianni, Luca (1989): Grammatica italiana. Italiano comune e lingua letteraria. Torino: Utet.

Simone, Raffaele (1995): Iconic Aspects of Syntax. In: Simone, Raffaele (Hg.): Iconicity in language. Amsterdam: Benjamins. S. 153-169. (= Current Issues in Linguistic Theory 110).

Sternefeld, Wolfgang (2008/2009): Syntax. Eine morphologisch motivierte generative Beschreibung des Deutschen. 2 Bde. 3., überarb. Aufl. Tübingen: Stauffenburg. (= Stauffenburg Linguistik 31).

Sweetser, Eve (1990): From etymology to pragmatics: Metaphorical and cultural aspects of semantic structure. Cambridge: Cambridge University Press. (= Cambridge Studies in Linguistics 54).

Traugott, Elizabeth Closs (1989): On the rise of epistemic meanings in English: An example of subjectification in semantic change. In: Language 65, S. 31-55.

Traugott, Elizabeth Closs/Dasher, Richard B. (2002): Regularity in semantic change. Cambridge: Cambridge University Press. (= Cambridge Studies in Linguistics 97).

Traugott, Elizabeth Closs/König, Ekkehard (1991): The semantics-pragmatics of grammaticalization revisited. In: Traugott, Elizabeth Closs/Heine, Bernd (Hg.): Approaches to grammaticalization. Bd. 1: Focus 
on theoretical and methological issues. Amsterdam: Benjamins. S. 189-218. (= Typological Studies in Language 19).

Uhmann, Susanne (1998): Verbstellungsvariation in weil-Sätzen: Lexikalische Differenzierungen mit grammatischen Folgen. In: Zeitschrift für Sprachwissenschaft 17, S. 92-139.

Wegener, Heide (1999): Syntaxwandel und Degrammatikalisierung im heutigen Deutsch? Noch einmal zu weil-Verbzweit. In: Deutsche Sprache 27, S. 3-26.

Wöllstein, Angelika (2010): Topologisches Satzmodell. Heidelberg: Winter. (= Kurze Einführungen in die germanistische Linguistik 8).

Zifonun, Gisela/Hoffmann, Ludger/Strecker, Bruno et al. (1997): Grammatik der deutschen Sprache. 3 Bde. Berlin/New York: De Gruyter. (= Schriften des Instituts für Deutsche Sprache 7).

\author{
Miriam Ravetto \\ Dipartimento di Studi Umanistici \\ Università del Piemonte Orientale \\ Piazza S. Eusebio, 5 \\ 13100 , Vercelli \\ Italien \\ E-Mail: miriam.ravetto@uniupo.it \\ Prof. Dr. Hardarik Blühdorn \\ Institut für Deutsche Sprache \\ Postfach 101621 \\ 68016 Mannheim \\ E-Mail: bluehdorn@ids-mannheim.de
}

